\title{
Coronary artery surgery and myxoedema
}

\author{
S J KIRK, * H O O'KANE, * P MORTON $\dagger$ \\ From the ${ }^{\star}$ Department of Cardiac Surgery, Royal Victoria Hospital, and $†$ Department of Cardiology, Belfast \\ City Hospital, Belfast
}

SUMMARY A patient with a preoperative diagnosis of myxoedema had triple vessel coronary artery bypass surgery. The perioperative and postoperative course was essentially uneventful. The most appropriate management of a patient with coronary artery disease who has concomitant myxoedema is first to improve myocardial blood flow by relieving coronary obstruction and then to treat the thyroid deficiency.

Management of patients with concomitant myxoedema and coronary artery disease is difficult. ${ }^{1}$ In 1938 Smyth described the clinical dilemmas associated with providing adequate thyroid hormone replacement therapy in such patients. ${ }^{2}$ Thyroxine supplementation commonly increases anginal symptoms, and progression to myocardial infarction has been reported. ${ }^{3}$ This is a particular problem when the myocardial disease is considered suitable for coronary artery revascularisation. Paine et al reported one death in six patients after attempted preoperative hormone replacement. ${ }^{1}$ To date, there has not been a large study of the diagnosis and subsequent management of hypothyroid patients with chest pain. In patients with myxoedema coronary artery disease tends to be more extensive ${ }^{4}$ than usual and these patients require detailed and careful investigation.

We present a case report which we believe lends support to a specific and useful form of management by adequate investigation followed by coronary artery revascularisation and postoperative thyroid hormone replacement therapy.

\section{Case report}

A 35 year old man was admitted to the cardiac surgery unit for myocardial revascularisation. He gave a three year history of increasing angina pectoris with recent exacerbation making him housebound. He was now experiencing rest and nocturnal pain and further questioning revealed recent weight gain $(20 \mathrm{lb}(10 \mathrm{~kg}))$ and cold intolerance. Angio-

Requests for reprints to Mr H O O'Kane, FRCS, Department of Cardiac Surgery, Royal Victoria Hospital, Grosvenor Road, Belfast BT12 6BA. graphy, a year before, had demonstrated severe triple vessel disease with $\geqslant 70 \%$ stenosis of the marginal circumflex and left anterior descending coronary arteries, complete blockage of the right coronary artery, and moderate distal disease.

He was obese (weight $14 \mathrm{st} 4 \mathrm{lb}(90 \mathrm{~kg})$, height $5 \mathrm{ft}$ 10 in $(178 \mathrm{~cm}))$. The pulse rate was 80 beats per minute and regular. His blood pressure was $100 / 60 \mathrm{~mm} \mathrm{Hg}$ prone, and heart sounds were normal. The jugular venous pressure was not elevated, there was no peripheral oedema, and his chest was clear. He had normal distribution of body hair and no palpable masses in neck or abdomen. Neurological examination suggested sustained ankle jerks.

Routine preoperative investigations were performed: haemoglobin $(14.6 \mathrm{~g} / \mathrm{dl})$; mean corpuscular volume ( $120 \mathrm{fl}$ (normal range 84-98)); normal platelet and leucocyte counts. Although creatine kinase was raised $(100 \mathrm{U} / 1$ (normal range $30-140 \mathrm{U} / \mathrm{l})$ ), the concentration of the isoenzyme creatine kinase was within normal limits. The electrocardiogram showed low voltages with widespread $T$ wave flattening and minimal ST depression in chest leads V4 to V6. There were no specific changes suggestive of myocardial infarction.

We thought that the initial haematological and biochemical findings together with the clinical and electrocardiographic findings suggested a diagnosis of myxoedema. Thyroid function tests were performed. These showed a free thyroxine value of $4 \mathrm{pmol} / 1$ (normal range 9-26), thyroid stimulating hormone $33 \mathrm{mU} / 1$ (normal range $0-5$ ). The diagnosis of myxoedema was confirmed immediately before operation. The operation was performed as planned with reverse saphenous vein grafting into the three main vessels; each graft required endarterectomy.

Although the procedure was carried out under controlled hypothermia the patient's temperature 
was $37.5^{\circ} \mathrm{C}$ when he came off bypass. The patient's postoperative course was essentially uneventful; however, he did require dopamine support ( $5 \mu \mathrm{g} / \mathrm{kg} / \mathrm{min}$ in reducing dosage) for twelve hours after operation. His temperature fell to $35 \cdot 3^{\circ} \mathrm{C}$ when he returned to the recovery ward. It remained at this level for 24 hours and was not normal until $\mathbf{4 8}$ hours after operation. Twenty four hours after operation, he was extubated and was started on L-thyroxine $25 \mu \mathrm{g} /$ day; the dose was gradually increased over ten days to $100 \mu \mathrm{g} /$ day.

He was discharged 14 days after operation and remained well and symptom free until four months after operation when anginal symptoms returned. At this stage his haemoglobin was $6.0 \mathrm{~g} / \mathrm{dl}$ and mean corpuscular volume was $98 \mathrm{fl}$; serum vitamin $B_{12}$ was at the lower limit of normal and serum folate at the upper limit of normal. Bone marrow examination revealed gross megaloblastic change at all stages of erythropoiesis. Pernicious anaemia was suspected; this diagnosis was confirmed by a positive Schilling test. Hydroxocobalamin treatment was started. After the haemoglobin concentration had returned to normal, the anginal symptoms regressed significantly but did not disappear. He continued to be biochemically and clinically euthyroid.

\section{Discussion}

In patients with severe and, in particular, unstable angina thyroid replacement therapy can exacerbate symptoms by increasing the heart rate, myocardial contractility, and myocardial oxygen consumption. Thus patients with angina and myxoedema often end up with continuing angina and inadequately treated myxoedema. ${ }^{1}$ Coronary artery bypass surgery may provide a more rational therapeutic approach to this combination of diseases because after successful coronary revascularisation the myxoedematous patient may be better able to tolerate adequate doses of thyroid preparations. ${ }^{134} \mathrm{We}$ believe that our experience with the present case supports this approach.

Our patient presented with clinically unstable angina and clinical and laboratory findings suggestive of myxoedema. Angiography indicated that he was a suitable, but not ideal, candidate for a revascularisation procedure (moderate "distal disease" was demonstrated). It was clear that he was not going to be able to withstand full hormone replacement therapy because he was profoundly hypothyroid and yet had rest pain on maximal antianginal treatment. With this in mind, and in view of the potential inadequacies of the available medical management, we decided to proceed to bypass surgery.

Operation in myxoedematous patients is more hazardous because of the increased risks of infection, cardiovascular instability and collapse, respiratory depression, paralytic ileus, and of the development of an inappropriate antidiuretic hormone-like syndrome. In addition body tissue is more friable. These potential problems must be weighed against the real danger of the complications associated with medical management. Once surgery is decided upon, the question of preoperative thyroid hormone replacement therapy must be faced and the conflicting evidence analysed.

Nelson et al reported two patients with myxoedema who underwent coronary artery bypass surgery; one did not have thyroid hormone supplementation before operation. ${ }^{4}$ His postoperative course was complicated by an inappropriate antidiuretic hormone-like syndrome, paralytic ileus, and infection. The second patient was given a small daily dose of $10 \mu \mathrm{g}$ thyroxine before operation and did well after operation on a dose of $200 \mu \mathrm{g}$ of Lthyroxine. The experience of Paine et al, however, suggests that thyroid replacement is not necessary before operation (only one of his series of six patients had a complicated course) and that thyroxine should be introduced gradually after operation with regular assessment of electrocardiograms and serum concentrations of thyroid stimulating hormone. Myerowitz et al found no noticeable improvement in haemodynamic function (cardiac index, left ventricular index, oxygen consumption) in 10 patients treated with small doses of thyroxine before operation. ${ }^{3}$ They therefore concluded that preoperative treatment offers no clinical or haemodynamic benefit.

The result in our patient supports the view that coronary artery surgery is not contraindicated in cases of untreated myxoedema and that revascularisation can be performed safely in such cases before thyroid replacement therapy, and indeed is the regimen of choice. Mild angina has persisted in our patient although it has become more stable. Because he remains euthyroid we believe that extensive distal disease is the main reason for the persistence of angina.

\section{References}

1 Paine TD, Rodgers WJ, Baxley WA, Russell RD. Coronary artery surgery in patients with incapacitating angina pectoris and myxoedema. Am J Cardiol 1977;40:226-31.

2 Smyth CJ. Angina pectoris and myocardial infarction as complication of myxoedema with special reference to the danger of treatment with thyroid preparations. Am Heart J 1938;15:552-660.

3 Myerowitz PD, Kamienski RW, Swanson DK, Chopra PS. Diagnosis and management of the hypothyroid patient with chest pain. J Thorac Cardiovasc Surg 1983;86:57-60.

4 Nelson JC, Palmer FJ, Bowyer AF. The successful treatment of myxoedema and coronary artery disease in patients intolerant of thyroid hormone. Med Arts Sci 1974;28:15-22. 